\title{
Evaluation of Dexterity and Eye Hand Co-ordination in Garment Industry Workers
}

\author{
Manasi Desai and Shruti Shah
}

\begin{abstract}
Aim: The aim of the study was to evaluate dexterity and eye hand coordination in garment industry workers Method: The study was conducted among 142 garment worker who fulfilled the inclusion criteria.The Purdue pegboard test was used for evaluation of dexterity and eye hand coordination in this population along with self-made questionnaire for demographic detail. Results and Conclusion: Based on the results of the study conducted among 142 garment workers, dexterity and eye hand coordination was found better in dominant hand as compared to non-dominant hand in both genders. In both hands dexterity task, age group of 26 to 35 years inboth genders was found better. Slight age related decline was seen in dexterity and eye handcoordination in the age group 36 to 45 years as compared to other ages. The dexterity and eye hand coordination values in these workers were similar to normative value in normal population.
\end{abstract}

\author{
Manasi Desai \\ Assistant Professor \\ D.Y. Patil School of Physiotherapy \\ D.Y. Patil University \\ Nerul, Navi Mumbai,India \\ E-mail: manasind11@gmail.com \\ Shruti Shah \\ B.P.Th. \\ D.Y. Patil School of Physiotherapy \\ D.Y. Patil University \\ Nerul, Navi Mumbai,India \\ E-mail:sdrshruti@gmail.com
}

Key Words: Purdue Pegboard, Dominant hand, Non-Dominant hand, Assembly, Eye hand Coordination and Dexterity, Physiotherapy

DOI: $10.18376 / j e s p / 2020 / v 16 / i 1 / 154132$

\section{Introduction}

German-born engineer, Charles Fredrick Wiesenthal working in England was awarded the first British patent for a mechanical device to aid the art of sewing, in 1755. His invention comprised of a double pointed needle with an eye at one end (William 2019). India is one of the world's largest producers in textile and garment industry (Sateeh et al., 2015). India has a very fast growing economy, $48 \%$ of the population in urban areas are highest wealth quintile, while only $7 \%$ of rural population is in the highest wealth quintile (Govt of India 2007). In developing countries great efforts are directed towards development of small scale industries. According to WHO (1997), over 1000 million people worldwide are employed in small scale industries. The 'Garment' industry is an unorganized sector, mostly run by private establishment. It has provided ample scope of employment to people from low socioeconomic status involving both men and women. In India such industries are considered to provide more opportunity to women as compared to man (Bandyopadhyay 2012). The small and medium scale enterprises (SMEs) are much greater in number in Indian economy and these are the places where work posture analysis is mostly neglected. Repetitive processes and manual material handling are the major problems in the hand tool manufacturing (Singh 2010). In India, the readymade garment industry had its beginning 


\section{Journal of Exercise Science \& Physiotherapy Vol. 16 No. 1 (January to June) 2020 ISSN: 0973-2020 (Print) $\mathrm{I}_{2} \mathrm{OR}$ Impact Factor $=6.850 \quad$ ISSN: 2454-6089 (Online)}

during the first half of the $20^{\text {th }}$ century and has witnessed impressive growth during the last four decades (Nag 1996).Today most of people spend their working days carrying out repetitive activities in awkward postures which may lead to injuries and muscle pain (Raichurkar 2017). The evolution of the hand has reached its highest degree of development in humans, and it has determined many of the unique functional and creative capabilities of the species. The hand aids as an important creative tool, an extension of intellect, a means of nonverbal communication, and a major sensory tactile organ. The quality of performance in work-related functioning,daily living skills and recreational activities is determined to a huge degree by hand function and manual dexterity (Carmeli et al., 2003). The hand is a receptor of much information from the environment and in everyday life all kinds of grips are of vital importance for ordinary activities in daily life (Kamieniarz et al., 2002). The hand is the maximum active and interactive part of the upper extremity. Hand dexterity is a term used to explain a range of different hand abilities and performances (Martin et al., 2015). Eye-hand coordination plays an essential role in adaptation as it's a complex psychomotor skill, which involves synergistic action of sensory functions (exteroceptive and interoceptive) and motor function, resulting in providing informational and energy parameters of the movement (Vasilica et al., 2012). Eye hand coordination refers to the synchronization of movements of the hand to visual stimuli. Skilful manipulation of an object by hands often involves reach, grasp, carry, release, and in hand manipulation and bimanual coordination.It depends on powerful, distributed neural networks and includes two concepts of speed and accuracy (Nandgaonkar and Hemant.2002). Manual dexterity is defined as the ability to integrate precision and speed with finely coordinated movements of the arm, hand and fingers and it also refers as the ability to use one's hands' or the ability to manipulate objects with the hands:' Backman. Mackie. and Harris' define dexterity as the tine voluntary movements used to manipulate small object during a specific task (Edeer et al.,2005; Desrosiers et al., 1994).During hand rehabilitation, dexterity testing is frequently used to assess manual function ${ }^{17}$ Manual dexterity is often evaluated in rehabilitation because of its contribution to upper limb performance and to individual functional independence (Desrosiers et al., 1994). Joseph Tiffin, Ph.D. industrial psychologist, Purdue university (1948), first develop Purdue pegboard test .The Purdue Pegboard is a test of dexterity that was designed to aid in selecting adults applying for industrial jobs such as assembly, packing, functioning machines, and other manual jobs . It consists of four subtests: right hand, left hand, both hands, and assembly. There is general agreement in the literature that there is more than one type of dexterity. Henry further hypothesized that motor abilities were specific to each particular task. Current therapy practice, however, is more consistent with the position taken by Fleishman, who identified 11 psychomotor abilities that he believed were the underlying bases of skilled performance. Five of these abilities were related to dexterity: wrist-finger speed, finger dexterity, speed of arm movement, manual dexterity, and aiming. The other six abilities were control precision, multi limb coordination ,response orientation ,reaction time, rate control, arm hand steadiness. The examiner's manual suggests that the Purdue Pegboard measures two types of dexterity, "one involving gross movement of hands, fingers, and arms and the other involving primarily what might be called 'fingertip' dexterity" (Mathiowetz, et al., 1986). The advantage of Purdue pegboard test are (a) Easy to administer and Measures dexterity as well as fine motor coordination. (b) It also examinees eye hand co-ordination. (c) It can be used to test hand function in neurological, musculoskeletal \& developmental disorders (Desai et al., 2006). In a 1958 pilot study, Siegel and Hirschhorn reported normative data on 100 male adolescents aged 12 to 18 years. Although they concluded that adolescents over 15 years of age could be compared with the adult norms, they suggested that more research with a larger number of subjects be done (Mathiowetz, et al., 1986). A review of literature has revealed that the Purdue Pegboard test has been used to evaluate dexterity and eye hand co-ordination of workers from garment industry.However there is 


\section{Journal of Exercise Science \& Physiotherapy Vol. 16 No. 1 (January to June) 2020 \\ ISSN: 0973-2020 (Print) I IOR Impact Factor $=6.850 \quad$ ISSN: 2454-6089 (Online)}

a lack of such studies in the workers from various industrial population. Hence, the aim of the present study was to evaluate the dexterity and eye hand co-ordination of garment industry workers.

\section{Materials and Method}

The study was cross-sectional prospective and carried out over a period of 6 months and one hundred forty two $(\mathrm{N}=142)$ participants were included in this study. Garment industry workers who were willing to participate in this study between the age group of 18 to 50 years were included in the evaluation. Participants who were unwilling to participate in the study or who had neurological /musculoskeletal impairment affecting their upper limb were excluded from the study. The study was approved by the Institutional Ethics and Research Committee at D.Y.Patil , School of Physiotherapy. A written consent was obtained from the participants before the study and their confidentiality was ensured. After collecting the demographic information, Dexterity and Eye hand coordination was assessed using Purdue Pegboard test. Three trials were done. The obtained data was statistically analysed and results were obtained.

\section{Results}

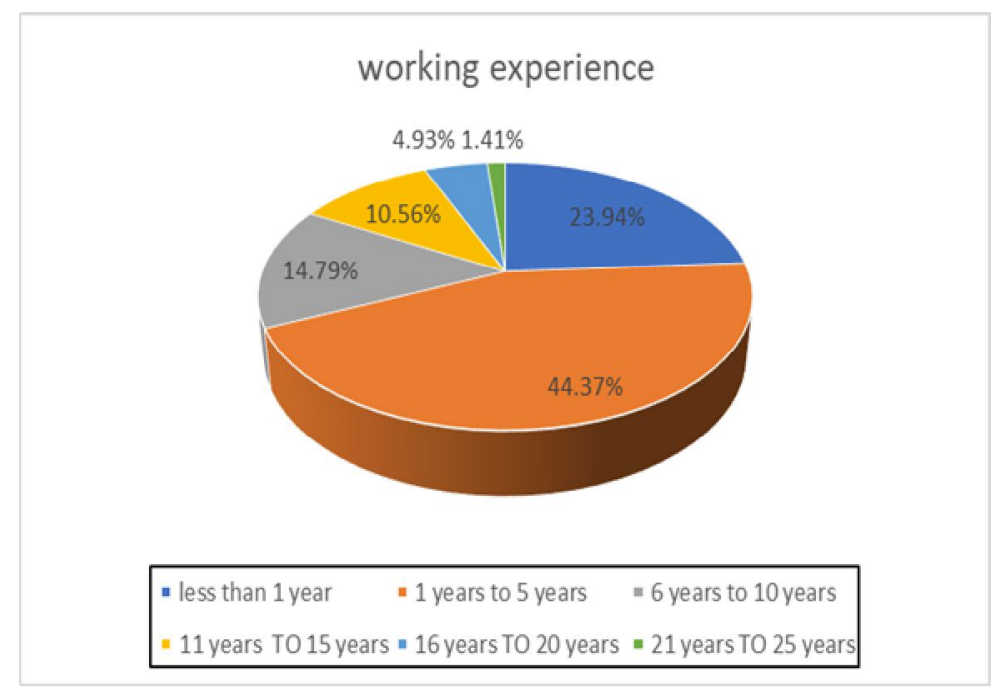

Figure 1. Work experience of the subjects

Figure 1 show the work experience of the subjects and it was found that out 142 garment workers, maximum workers had an experience between 1 year to 5 years $(44.37 \%)$ and minimum had an experience between 21 year to 25 years $(1.41 \%)$. 


\section{Journal of Exercise Science \& Physiotherapy Vol. 16 No. 1 (January to June) 2020 ISSN: 0973-2020 (Print) $\quad \mathrm{I}_{2} \mathrm{OR}$ Impact Factor $=6.850 \quad$ ISSN: 2454-6089 (Online)}

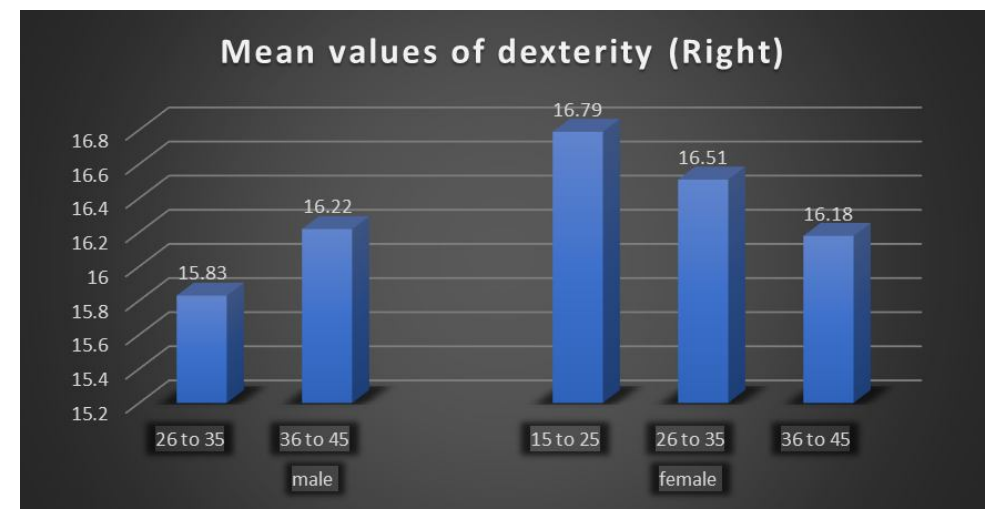

Figure 2. Right hand dexterity

Figure 2 shows average value of right hand dexterity in both genders. In males, maximum dexterity was seen in age of 36 to 45 years. In females, dexterity was slightly higher in age group of 15 to 25 years as compare to other age groups.

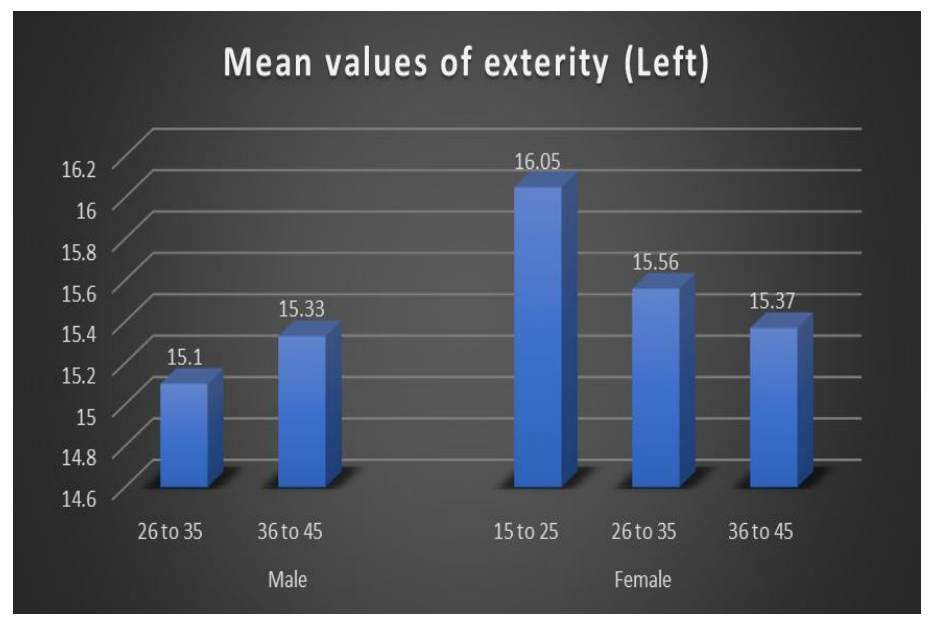

Figure 3. Left hand dexterity

Figure 3 shows mean values of left hand dexterity in both genders. In males, dexterity was slightly higher in age group of 36 to 45 years. In females, dexterity was slightly higher in age group of 15 to 25 years as compare to other age groups.

Figure 4 shows average values of both hand dexterity in both genders. In both males and females, the dexterity values were seen slightly higher in age group of 26 to 35 years as compared to other age groups. 


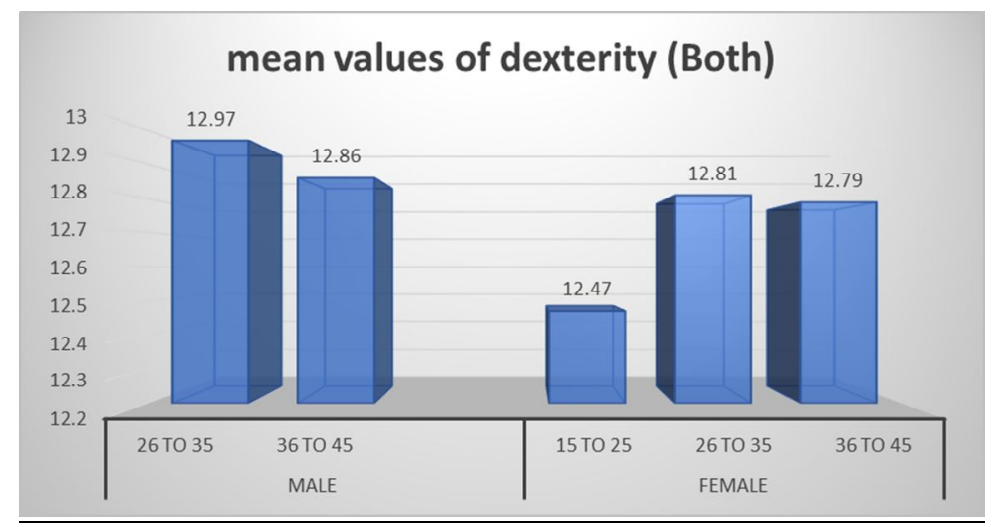

Figure 4. Both hands dexterity

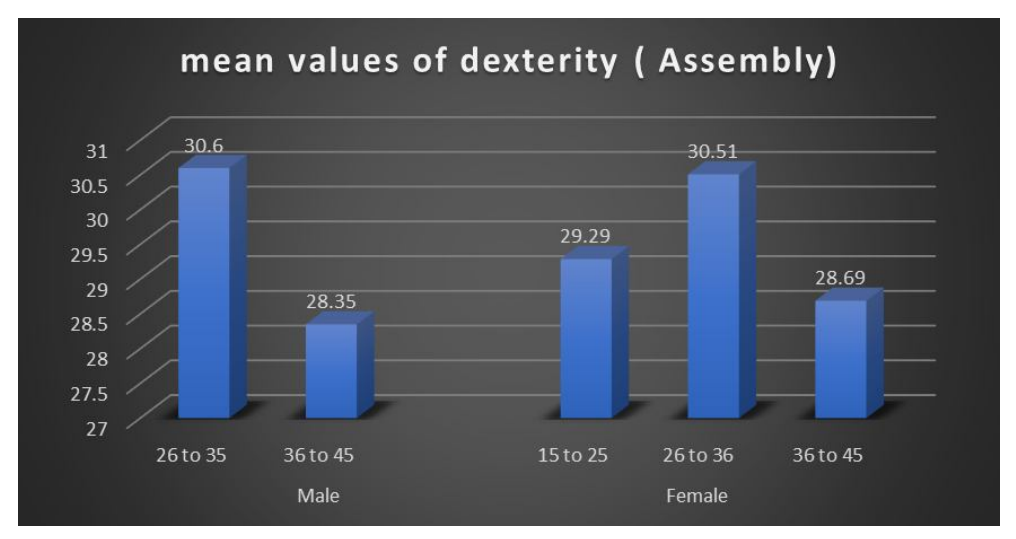

Figure 5. Mean values of Assembly in both genders

Figure 5 shows mean values of Assembly in both genders. In males and females, the dexterity was seen slightly higher in age group of 26 to 35 years as compared to other age groups.

\section{Discussion}

The present study was undertaken to evaluate the dexterity and eye hand coordination of 142 garment workers from the industry. Majority subjects were females (74\%), as compared to males $(26 \%)$. The maximum workers had a work experience of 1 to 5 years and least had an experience of 21 to 25 years (Figure 1). In the present study all the subject had right-handed dominance with daily working of 8 hours with rest duration of one hour. They worked for 6 days per week. The first objective of the study was to evaluate the dexterity and eye hand coordination in the garment workers using Purdue pegboard. The Purdue Pegboard is a test of uni - and bi-manual dexterity. 


\section{Journal of Exercise Science \& Physiotherapy Vol. 16 No. 1 (January to June) 2020 ISSN: 0973-2020 (Print) $\quad \mathrm{I}_{2} \mathrm{OR}$ Impact Factor $=6.850 \quad$ ISSN: 2454-6089 (Online)}

The evolution of the hand has reached its highest degree of development in humans, and it has determined many of the unique functional and creative capabilities of the species. It is clear that common tasks involving precision dexterity, two-hand coordination, are needed to thread needles, open buttons on clothing, or fine-grip tasks (Carmeli et al., 2003). For right-handed and left handed dexterity, in females maximum mean value was obtained in the age group of 15 to 25 years, minimum mean value was in age group of 36 and 45 years. In males highest mean value was obtained in the age group of 36 and 45 years, whereas lowest mean value was in age group of 26 to 35 years ( Figure 2 and 3).Overall the mean for right hand dexterity was higher than left hand dexterity. From these above average values, it is clear that workers had better dexterity in dominant hand. Similar results were found in a study done by Walankar ,et al in Indian population stating that grip strength of dominant hand is greater than a nondominant hand (Walankar et al., 2016). When dexterity of both hands were assessed, in females maximum mean value was obtained in the age group of 26 to 35 years, whereas minimum mean value was in age group of 15 to 25 years. In males, highest average value was in the age group of 26 to 35 years, lowest average value was in age group of 36 to 45 years( Figure 4).During assembly, equal use of both hands is essential throughout operating the test. In both males and females, highest mean value was obtained in age group of 26 to 35 years, whereas lowest mean value was obtained in age 36 to 45 years (graph 5).

Thus, from our study we concluded that while performing bimanual dexterity and during assembly of the pegs, workers between the age group of 26 to 35 years (both male and female) performed betterthan other age groups. But it is also seen that, there is mild decrease in dexterity and eye hand coordination in group of 36 to 45 years during assembling. The relationship between increased age and reduced hand dexterity has been widely reported in the clinical and scientific literature.A common explanation is provided in the discussion of these papers stating that the relationship between increased age and reduced hand dexterity is likely caused by a decreased in muscle strength and mass (Martin et al., 2015). It was also noticed that in age group of 15 to 25 years females, the bimanual dexterity and eye hand coordination was lesser than other age groups .This could be because this worker were new for work in garment industry and were not trained for fine motor task as much as others. Therefore, suitable training program should be organized for the workers, as this will improve their dexterity and eye hand coordination and help in improve their productivity. Those with less experience have higher chances of getting injured which could affect the work and suitability for the job. It was also seen that females performed better than males on three out of four subtests. Similar results were found in the study done by Mathiowetz et al., (1986) in 14 to 19 years old stating that females performed better than males. Based on the work profile, it is clear that garment industry workers require high degree of dexterity and eye hand coordination as compare to general population. However the values obtained our study are similar to the average values for various age group in normal Indian population as obtained by Desai et al., (2006). These workers however have to perform more fine motor task than a normal population, therefore this demands of having higher values for a better job. The hand dexterity tasks of steadiness and line tracking both rely on a stable control of the arm and hand, and eye-hand coordination with visual guidance. ${ }^{14}$ Hand dexterity is closely related to one's occupation. Thus, there should be a target specific training program organized to increase their dexterity and eye hand coordination. This may improve their suitability for work and also help them to increase these work productivity.

\section{Conclusion}

Based on the results of the present study it was concluded that dexterity and eye hand coordination was found better in dominant hand as compared to non-dominant hand in both genders (males and females ). During both hands dexterity task, it was seen better in age group of 26 to 35 years in both genders and lowest in age group of 36 to 45 years in males and 15 to 25 years in females. In 


\section{Journal of Exercise Science \& Physiotherapy Vol. 16 No. 1 (January to June) 2020 \\ ISSN: 0973-2020 (Print) $\quad$ I $_{2}$ OR Impact Factor $=6.850 \quad$ ISSN: 2454-6089 (Online)}

assembly, slight age related decline was seen in dexterity and eye hand coordination in the age group 36 to 45 years as compared to 26 to 35 years.

\section{Reference(s)}

Bandyopadhyay L.2012.Musculoskeletal and Other Health Problems in Workers of Small Scale Garment Industry - An Experience from An Urban Slum, Kolkata. IOSR Journal of Dental and Medical Sciences, 2(6):23-8.

Carmeli E, Patish H, Coleman R. 2003.The Aging Hand. The Journals of Gerontology: Series A. 2003;58(2).

Desai K. Kene K. Doshi M et al.2006.Normative data of purdue pegboard on Indian Population. The Indian Journal of Occupational Therapy :37(3):69-72.

Desrosiers J, Bravo G, HChert R, Dutil E, Mercier I,.1994.Validation of the Box and Block Test as a measure of dexterity of elderly people: reliability validity, and norms studies. Arch Phys Med Rehabil:75:751s.

Edeer, Ayse \& Tulum, Zeliha \& Pinar, Lamia \& Başkurt, Ferdi.2005. Comparison of Pressure Pain Threshold, Grip Strength, Dexterity and Touch Pressure of Dominant and Non-Dominant Hands within and Between Right- and Left-Handed Subjects. Journal of Korean medical science. 19. 874-8. 10.3346/jkms.2004.19.6.87\} .

Govt of India (2007). National Family Health Survey - 3, 2005-2006; 1: Ministry of Health and Family Welfare.

Kamieniarz, M \& Stryła, Wanda \& Kowalska, P \& Kamieniarz, G.2002.Assessment of the children manual dexterity and hand grip strength: test-retest reliability study. Computational Methods in Science and Technology. 8. 10.12921/cmst.2002.0.01.69-78.

Martin JA, Ramsay J, Hughes C, Peters DM, Edwards MG.2015.Age and Grip Strength Predict Hand Dexterity in Adults. Plos One ;10(2).

Mathiowetz, V., Rogers, S. L., Dowe-Keval, M., Donahoe, L., \& Reline's, C.1986. The Purdue Pegboard: Norms for 14- to 19- year-olds. The American Journal of Occupational Therapy, 40, 174-179.

Nag A.1996.Women in Industry - Repetitive Work and Postural Stress. In: Nag P K, (Ed). Ergonomics and Work Design - Emerging Issues in Organisational Sciences. New Age International Pvt Ltd: New Delhi.

Hemant P. Nandgaonkar..2002. Rivet \& Eyelet Deftness Test (Red Test): A Measure for Hand Dexterity. The Indian Journal of Occupational Therapy,IJOT : Vol. XXXIV: No. 1,April-July 2002,pp.13-22.

Raichurkar, Pramod.2017. Textile apparel industry: A responsibility towards manpower. Colourage. May 2017, 38 to 46. https://www.researchgate.net/publication/317169585

Sateeh Kulkarni, ASA \& Associates LLP.2015. A Brief Report on Textile Industry In India. July 2015.

Singh, Lakhwinder Pal. 2010."Work posture assessment in forging industry : An exploratory study in India ". International Journal of Advanced Engineering Technology, IJAET, Vol.I, Issue III,Oct.-Dec.,358366.

Vasilica Grigore, Georgeta Mitrache, Radu Predoiu, and Remus Roşca.2012. Characteristic of instrumental movements - eye hand coordination in sports. Procedia - Social and Behavioral Sciences, vol. 33, 2012. doi:10.1016/j.sbspro.2012.01.

Walankar P, Verma C, Mehta A.2016. Study of hand grip strength in Indian population. Int J Health Sci Res. 6(11):162-166.

WHO.1997.Occupational Health: The work place. Health and environment in sustainable development. Geneva; http://www.who.int/pen/occupational health/ occupational health2.htm

William Trammell Snyder. "Charles Fredrick Wiesenthal (1726-1789): An Appraisal of the Medical Pioneer of Baltimore." pp.47-58. https://en.wikipedia.org/wiki/Charles_Fredrick_Wiesenthal._Retrieved 21 October 2019.

Conflict of Interest: None declared 